\title{
Tendência temporal da hanseníase em uma região de saúde do Ceará, 2001 a 2015
}

\author{
Temporal trend in leprosy in a health region of Ceará, 2001 to 2015 \\ La tendencia temporal de la lepra en una región de salud de Ceará, 2001 a 2015 \\ Amanda Luiza Marinho Feitosa ${ }^{1}$ (D), Francisco Wellington Dourado Júnior ${ }^{2}$ (D) Caroline Mary Gurgel Dias Florêncio $^{3}$ (D)
}

\section{RESUMO}

Objetivo: Descrever a tendência temporal da hanseníase e seus indicadores epidemiológicos em uma região de saúde do estado do Ceará, no período de 2001 a 2015. Métodos: Estudo ecológico de base temporal, realizado a partir de dados obtidos pelo Sistema Nacional de Agravos de Notificação (SINAN), referentes aos casos novos de hanseníase registrados durante o período de 2001 a 2015. Os dados foram analisados a partir do modelo de regressão por pontos de inflexão (Joinpoint). Resultados: Dos 1.151 casos notificados no período, verificou-se redução do coeficiente de detecção geral e em menores de 15 anos. Em relação à proporção de casos com grau 2 de incapacidade física, identificou-se uma tendência de aumento significativo (APC = 9,04; IC95\% 1,0-17,8), assim como nos casos multibacilares ( $A P C=2,89 ;$ IC95\% 0,3-5,5). Conclusão: Concluiu-se que há aumento no número de casos multibacilares e com grau 2 de incapacidade física, tornando-se necessário o fortalecimento das medidas de vigilância e controle da doença na região.

Palavras-Chave: Hanseníase; Notificação de doenças; Doenças Endêmicas; Doenças Negligenciadas; Estudos de Séries temporais.

\begin{abstract}
Objective: Describing the temporal trend of leprosy and its epidemiological indicators in a health region in the state of Ceará, from 2001 to 2015. Methods: Ecological study based on the indicators monitored using the Information System of Notifiable Diseases. Data were analyzed using the inflection point regression model (Joinpoint). Results: Of the 1,151 cases reported in the period, there was a reduction in the general detection coefficient and children under 15 years old. For proportions of cases with grade 2 physical disability, a significant increase (APC = 9.04; 95\% CI 1.0-17.8) was observed, as well as in multibacillary cases (APC $=2.89 ; 95 \%$ CI $0.3-5.5$ ). Conclusion: There is an increase in the number of multibacillary cases and with grade 2 of physical disability, making it necessary to strengthen the measures of surveillance and disease control in the region.
\end{abstract}

Keywords: Leprosy; Disease Notification; Endemic Diseases; Neglected Diseases; Time-series Studies.

\section{RESUMEN}

Objetivo: Describir una tendencia temporal de la lepra y sus indicadores epidemiológicos en una región de salud en el estado de Ceará, de 2001 a 2015. Métodos: Estudio ecológico basado en los indicadores monitoreados utilizando el Sistema de información de registro de notificaciones. Se utilizó el modelo de regresión del punto de inflexión (Joinpoint). Resultados: De los 1,151 casos reportados en el período, hubo una tendencia a la baja en el coeficiente de detección general y en niños menores de 15 años. Para la proporción de casos grado 2 de discapacidad física, la tendencia fue a un aumento significativo (APC $=9.04$; IC del 95\%: 1.0-17.8), así como en casos multibacilares (APC = 2.89; IC del 95\%: 0.3-5) Conclusión: se concluye que existe una tendencia a aumentar el número de casos multibacilares y con un grado 2 de discapacidad física, por lo que es necesario fortalecer las medidas de vigilancia de la enfermedad en la región.

Palabras claves: Lepra; Notificación de Enfermedades; Enfermedades Endémicas; Enfermedades Desatendidas; Estudios de Series Temporales.

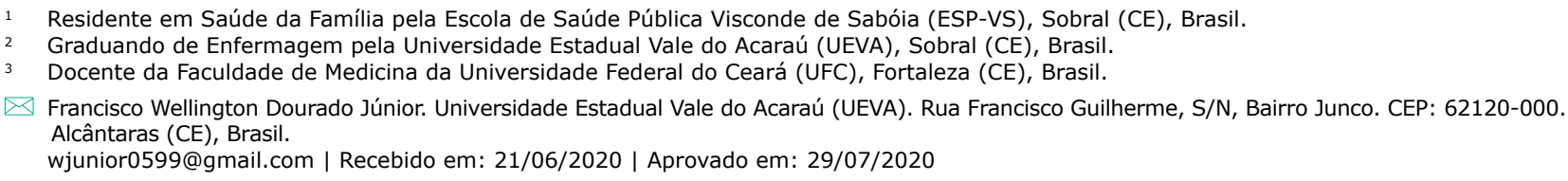




\section{INTRODUÇÃO}

A hanseníase é uma doença infectocontagiosa causada pela Mycobacterium leprae, que apesar das estratégias para o seu controle, continua como um grave problema de saúde pública, afetando milhares de pessoas anualmente. A doença se distribui de forma heterogênea no mundo, afetando principalmente países em desenvolvimento e quando não tratada precocemente, pode acarretar deficiências, deformidades e incapacidades físicas, fatos que complicam a morbidade, causam limitações na vida social e sofrimentos psicológicos ${ }^{1}$.

A prevalência da doença diminuiu globalmente, contudo, a detecção de casos novos permanece elevada em diferentes regiões do mundo. Em 2016, foram notificados 214.783 casos novos de hanseníase em 143 países, sendo a Índia responsável por 135.485 $(63,1 \%)$ desses casos, o Brasil por 25.218 casos $(11,7 \%)$ e a Indonésia por 16.826 casos $(7,8 \%)^{2}$.

Do ponto de vista global, o Brasil constitui um dos países prioritários para eliminação da hanseníase, apesar dos avanços obtidos na identificação de casos novos e tratamento dos mesmos, com consequente redução na incidência. Percebe-se uma considerável queda nos índices de casos novos no país, que em 2009 apresentava 37.610 novos casos da doença e em 2017, esse montante foi reduzido a 22.940 novos casos ${ }^{3}$. Apesar disso, a taxa de detecção de novos casos no país atingiu 1,2 por 10.000 habitantes, ainda considerada "muito alta" de acordo com os parâmetros estabelecidos pela OMS4.

Como estratégia de vigilância e controle da hanseníase no âmbito nacional, foram implementadas políticas de tratamento ambulatorial, campanhas e diretrizes para direcionar os serviços de saúde em todo o país. Por intermédio do Plano Nacional de Eliminação da Hanseníase, os estados e municípios promoveram ações como oficialização da implantação da poliquimioterapia e diagnóstico e tratamento para todos os casos novos esperados, além disso, recebeu ênfase o diagnóstico precoce (objetivo de diagnosticar $90 \%$ dos casos novos antes do aparecimento de deformidades físicas), a promoção de alta por cura em $80 \%$ dos casos que iniciaram o tratamento e a redução da taxa de prevalência em 15\% a $20 \%$ ao ano ${ }^{5}$.
Para potencializar o controle da hanseníase, é necessário, porém, considerar os fatores que podem estar associados ao surgimento de novos casos da doença, como situação socioeconômica, faixa etária, escolaridade, gênero masculino e residência rural ${ }^{5}$.

Nesse contexto, é necessário que a Atenção Primária à Saúde assuma o protagonismo no controle da doença, bem como adote ações eficazes de prevenção, identificação de novos casos e acompanhamento daqueles que são diagnosticados, a fim de garantir a eficácia do tratamento e, assim, conter a cadeia de transmissão. O uso de instrumentos adequados e que permitam a análise dos fatores que permeiam o contexto da incidência da doença facilita também o planejamento das ações para o serviço primário, repercutindo diretamente na qualidade deste 6 .

Outro fator a ser considerado na assistência à saúde de pacientes com hanseníase são as incapacidades funcionais severas que podem ser ocasionadas pela doença, principalmente quando se trata do público idoso ${ }^{7}$, tendo em vista que mesmo após o término da poliquimioterapia, tais incapacidades podem surgir e se agravarem, caso os pacientes não recebam uma assistência à saúde de qualidade com a devida avaliação neurológica e física de profissionais capacitados ${ }^{8}$.

Logo, analisar o risco de transmissão hansênica e influência da situação epidemiológica em regiões de saúde corrobora para análise da incidência da doença e sua distribuição temporal em áreas geográficas determinadas e circunvizinhas, a fim de traçar intervenções específicas para o seu controle, principalmente nas regiões de fronteiras, onde há carências de estudos e fragilidade em vigiar a migração de populações entre as cidades ${ }^{9}$.

Dessa forma, o presente estudo tem como objetivo analisar a tendência temporal da hanseníase e seus indicadores epidemiológicos na $15^{a}$ Coordenadoria Regional de Saúde do estado do Ceará, no período de 2001 a 2015.

\section{MÉTODOS}

Trata-se de um estudo ecológico de séries temporais para caracterização da situação epidemiológica 
da hanseníase na $15^{a}$ Coordenadoria Regional de Saúde (CRES) do estado do Ceará, utilizando dados secundários oriundos do Sistema de Informação de Agravos de Notificação (SINAN), referente aos anos de 2001 a 2015.

O estudo foi conduzido no Estado do Ceará, situado na região Nordeste que tem uma população estimada de 9.020.46 habitantes e uma densidade demográfica de 56,76 habitantes por $\mathrm{km}^{2}$. Em sua divisão político-administrativa, possui 184 municípios, tendo Fortaleza como a capital ${ }^{10}$.

Atualmente, o Ceará possui cinco macrorregiões de saúde - Fortaleza, Sobral, Cariri, Sertão Central e Litoral Leste/Jaguaribe - sendo que estas estão subdivididas em 22 Coordenadorias Regionais de Saúde (CRES), que tem por finalidade orientar, planejar, acompanhar, avaliar e monitorar as atividades e ações de saúde em âmbito regional ${ }^{11}$.

Optou-se em analisar a $15^{a}$ Coordenadoria Regional de Saúde (CRES) pela ausência de estudos que analisassem a referida região e por ser o local em que a pesquisadora atuava enquanto graduanda de enfermagem. Tal região é composta pelos seguintes municípios: Ipaporanga, Ararendá, Poranga, Ipueiras, Nova Russas, Tamboril, Monsenhor Tabosa, Independência, Novo Oriente, Quiterianópolis e Crateús, município sentinela para esta CRES.

A população foi constituída por todos os casos novos de hanseníase em qualquer faixa etária, devidamente notificados através do SINAN da região proposta no estudo. Foram excluídos do estudo os dados que não se encontraram devidamente notificados no sistema, estando incompletos, tais como: ausência da forma clínica da hanseníase, ou aspectos demográficos e sociais.

Para caracterização dos casos, foram obtidas as seguintes variáveis: sexo, faixa etária, classificação operacional, raça, forma clínica e grau de incapacidade no momento do diagnóstico. Para a definição do padrão epidemiológico da hanseníase da região, foram calculados os seguintes indicadores: coeficiente de detecção anual de casos novos (CN) na população geral por 100.000 habitantes (mede a força de morbidade, magnitude e tendência de endemia), coeficiente de detecção anual de $\mathrm{CN}$ em crianças menores de 15 anos de idade por 100.000 habitantes (mede a força da transmissão recente da endemia e sua tendência), coeficiente de casos de hanseníase em qualquer faixa etária com grau 2 de incapacidade física (GIF 2) no momento do diagnóstico por 100.000 habitantes (deformidades causadas pela hanseníase na população geral, possibilitando compará-las com outras doenças incapacitantes) e, por fim, proporção de CN com classificação operacional multibacilar (MB) (indicando casos sob risco de desenvolver complicações e o correto reestabelecimento de poliquimioterapia $)^{12}$.

A fim de analisar a tendência temporal da doença, foi utilizado o modelo de regressão Joinpoint Regression Program (versão 4.0.4) que realiza o cálculo de incremento anual dos casos de recidiva do período total, 2001 a 2015. Este programa executa uma regressão linear segmentada para estimar a variação anual e percentual, identificar pontos em que há modificação da tendência.

O estudo respeita a Resolução do Conselho Nacional de Saúde, no 466, de 12 de outubro de 2012, e foi aprovado pelo Comitê de Ética em Pesquisa da Faculdade Princesa do Oeste-FPO (Parecer no 184/2018, 10 de setembro de 2018). Todos os dados utilizados neste artigo são de acesso e domínio público.

\section{RESULTADOS}

No recorte temporal de 2001 a 2015, foram notificados 1.151 casos novos de hanseníase na microrregião de Crateús, com maior proporção entre pessoas do gênero masculino sendo $53,77 \%(n=619)$, de cor parda sendo equivalente à $51,1 \%(n=588)$ e com idade superior a 15 anos representando $96,1 \%(n=1107)$ do total. A classificação operacional da doença foi predominantemente multibacilar 55,9\% $(n=640)$. Quanto à forma clínica, prevaleceu entre os casos notificados a indeterminada, com $28,93 \%$ $(n=296)$ e a forma dimorfa com $22,48 \%(n=230)$, seguidas da tuberculoide com $18,57 \%(n=190)$ e virchowiana representando $14,56 \% \quad(n=149)$. Vale ressaltar que $15,44 \%(n=158)$ dos casos não foram classificados nessa variável.

O coeficiente de detecção de $\mathrm{CN}$ geral em 2015 foi de 10 casos/100.000 habitantes. Em todo o período do estudo, a média desse indicador foi de aproximadamente 20,5 casos/ 100.000 habitantes e 
em 2008, foi registrado o pico mais elevado com 39 casos/100.000 habitantes (Figura 1). Ao longo da série histórica, a APC foi de -2,4 (IC95\% -5,6-1,2), indicando uma tendência decrescente (Tabela 1).

O coeficiente de detecção de $\mathrm{CN}$, em menores de 15 anos em 2014 foi de 16 casos/100.000 habitantes. Importante destacar que não foi possível considerar o ano de 2015 devido às informações do SINAN referentes ao ano não estarem consolidadas. O valor padrão da faixa etária em questão ao longo da série histórica foi de 2,73 casos $/ 100.000$ habitantes, e maior deteç̧ão foi em 2003 (6,58 casos/100.000 habitantes) (Figura 2). A análise de regressão por Joinpoint mostrou tendência decrescente da detecção em menores de 15 anos para a região de Crateús, com significância estatística (APC = 7,22; IC95\% -13,7 a -0,2) (Tabela 1).

A proporção média de casos com GIF 2 avaliada no momento do diagnóstico da hanseníase foi de $91,49 \%$ ( $n=1027)$. Essa proporção foi calculada a partir da média aritmética entre todos os coeficientes de casos novos de hanseníase com grau 2 de incapacidade que foram registrados ao longo da tendência temporal. Verificou-se também, elevação da proporção de CN com GIF 2 no momento do diagnóstico, de 0,27 casos por 100.000 habitantes em 2002, para 1,34 casos por 100.000 habitantes em 2015 (Figura 3).

Em 2011, identificou-se maior proporção de casos MB, representando $70,83 \%$ da média nos 15 anos da série, elevando-se de $43 \%$ em 2001 para 84,61\% em 2015 (Figura 4). A análise de regressão confirmou tendência de crescimento significante a partir de 2005 (APC = 2,89; IC95\% 0,3-5,5), exceto pelo abrupto declínio em 2008, cujas taxas de proporção atingiram o menor nível da série histórica, com $36 \%$. Tal fato pode ser considerado como instigante, visto que se trata do ano com maior índice de detecção de casos de hanseníase, mas o que apresenta menor proporção de casos na forma transmissível da doença (Tabela 1).

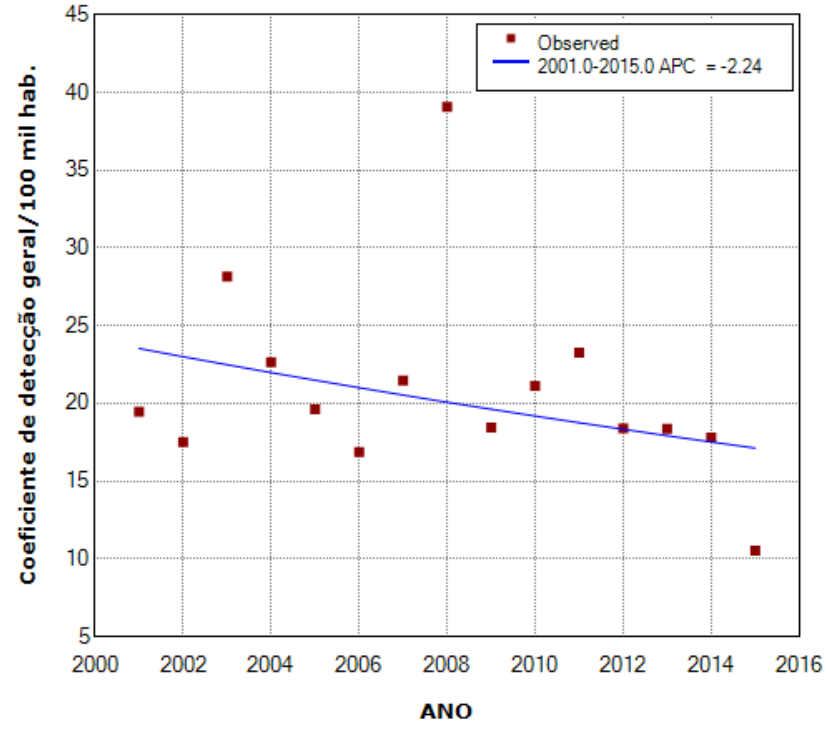

Figura 1. Série histórica do coeficiente de detecção geral de casos novos de hanseníase por 100.000 habitantes da $15^{\mathrm{a}}$ Coordenadoria Regional de Saúde, 2001-2015. Crateús-CE, 2020.

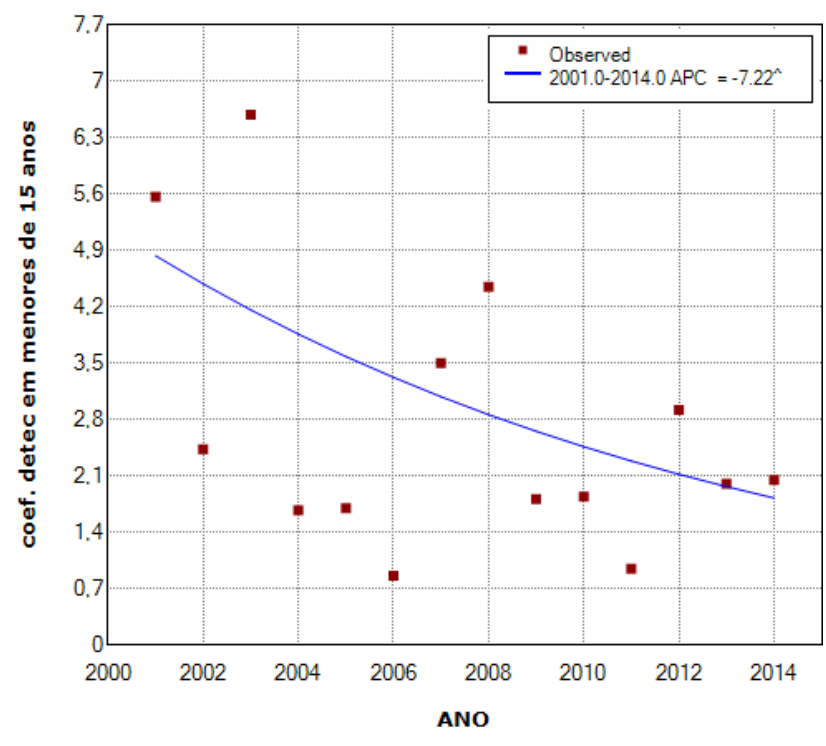

Figura 2. Série histórica do coeficiente de detecção em menores de 15 anos por 100.000 habitantes da $15^{\mathrm{a}}$ Coordenadoria Regional de Saúde, 2001-2015. Crateús-CE, 2020. 


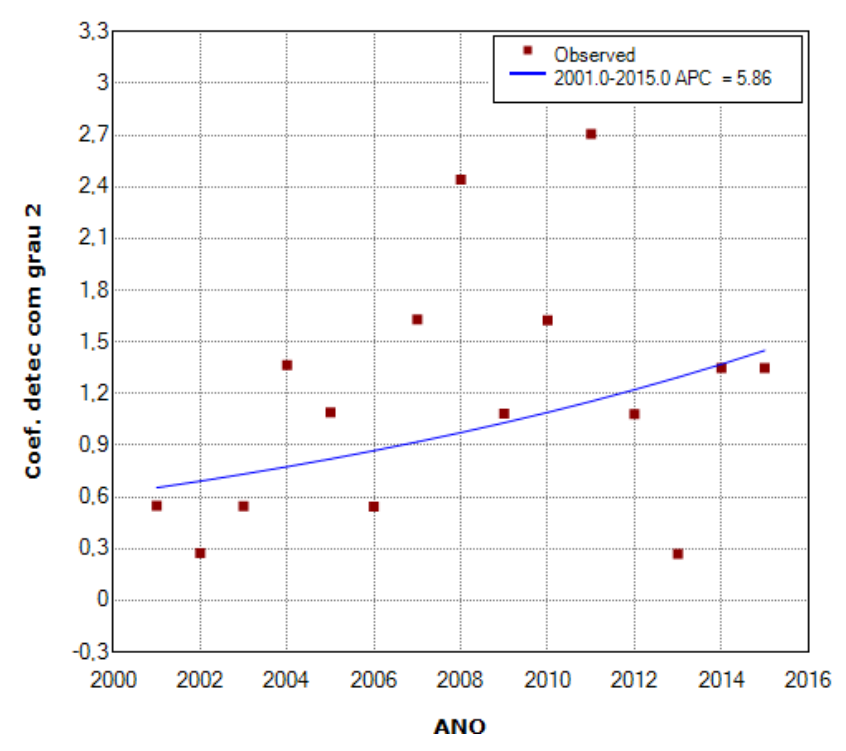

Figura 3. Série histórica do coeficiente de detecção de casos novos de hanseníase com grau 2 de incapacidade física no momento do diagnóstico por 100.000 habitantes da $15^{a}$ Coordenadoria Regional de Saúde, 2001-2015. Crateús-CE, 2020.

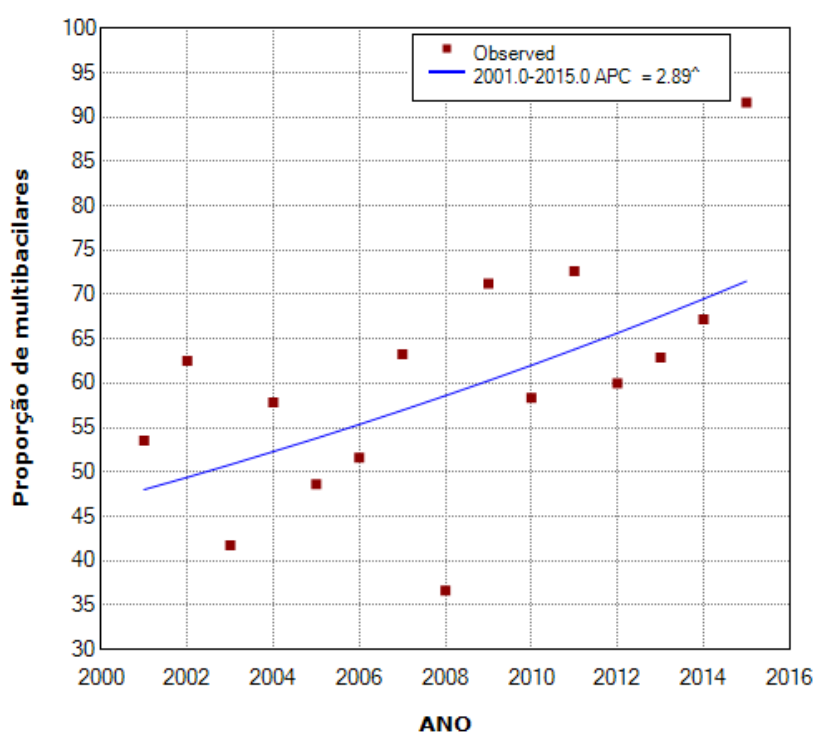

Figura 4. Série histórica da proporção de casos multibacilares de hanseníase da $15^{\mathrm{a}}$ Coordenadoria Regional de Saúde, 2001-2015. Crateús-CE, 2018.

\section{Tabela 1.}

Tendência temporal por pontos de inflexão (modelo Joinpoint) dos indicadores epidemiológicos e operacionais da hanseníase na $15^{a}$ Coordenadoria Regional de Saúde, 2001 a 2015. Crateús-CE, 2020.

\begin{tabular}{|c|c|c|c|}
\hline Indicador & APC & IC95\% & p-valor \\
\hline $\begin{array}{l}\text { Coeficiente de detecção de } \\
\text { casos novos }\end{array}$ & $-2,2$ & $\begin{array}{c}-5,6 a \\
1,2\end{array}$ & 0,185 \\
\hline $\begin{array}{l}\text { Coeficiente de detecção de } \\
\text { casos novos em menores de } \\
15 \text { anos }\end{array}$ & $-7,22$ & $\begin{array}{c}-13,7 \mathrm{a} \\
-0,2\end{array}$ & 0,044 \\
\hline $\begin{array}{l}\text { Coeficiente de detecção de } \\
\text { casos novos com GIF2 }\end{array}$ & 5,86 & $\begin{array}{l}-3,1 a \\
15,7\end{array}$ & 0,188 \\
\hline Proporção de casos < 15 anos & $-8,59$ & $\begin{array}{l}-14 a \\
-2,8\end{array}$ & 0,007 \\
\hline Proporção de casos com GIF2 & 9,04 & $\begin{array}{l}1,0 \text { a } \\
17,8\end{array}$ & 0,03 \\
\hline $\begin{array}{l}\text { Proporção de casos } \\
\text { multibacilares }\end{array}$ & 2,89 & $\begin{array}{c}0,3 a \\
5,5\end{array}$ & 0,028 \\
\hline Proporção de casos femininos & $-1,74$ & $\begin{array}{l}-3,3 a \\
-0,1\end{array}$ & 0,03 \\
\hline
\end{tabular}

\section{DISCUSSÃO}

O presente estudo apresenta, de forma sistemática, o contexto epidemiológico da hanseníase em uma série histórica extensa de 15 anos na $15^{a}$ Coordenadoria Regional de Saúde de Crateús e municípios limítrofes. A doença apresentou uma tendência decrescente e com padrões desiguais de expressão ao longo do tempo. Essas tendências, associadas à força de morbidade e de transmissão recente persistente, ao diagnóstico tardio e à provável endemia oculta pelo elevado percentual de casos multibacilares e casos com incapacidades visíveis, ampliam a relevância da hanseníase como problema de saúde pública na região. A análise por meio de uma série histórica permite uma melhor compreensão quanto à magnitude da endemia, tornando central o diagnóstico e tratamento oportuno com estratégias específicas para pacientes hansênicos.

A região de saúde em questão apresentou aumento do IDH na maior parcela dos seus 
municípios durante os anos de 2008 a $2010^{13}$ (IBGE). Associado a este fato, percebe-se que nesse período houve um declínio do número de casos de Hanseníase. Essa associação entre às condições de vida de uma determinada população e a frequência da hanseníase é reafirmada em um estudo realizado em Minas Gerais, que encontrou como resultado no primeiro período da pesquisa (1998 a 2005) a diminuição de 0,047 na taxa de detecção geral da doença com o aumento de 0,1 no valor do IDH dos municípios em $2000^{14}$.

No que concerne ao coeficiente de deteç̧ão de $\mathrm{CN}$, o pico mais elevado ocorreu em 2008, dando início a partir daí a um declínio desse coeficiente. No fim de 2008, a cobertura populacional atendida pelas equipes de atenção básica da região de saúde de Crateús atingiu 48,5\%, fato este que pode estar relacionado ao elevado pico no ano em questão ${ }^{15}$. Um estudo abordando o impacto da cobertura da Estratégia Saúde da Família (ESF) no controle da hanseníase evidenciou que a distribuição geográfica das taxas de deteç̧ão coincide com as áreas de maior vulnerabilidade social e incipiente cobertura dos serviços de ESF, que desempenha importante papel na criação de um planejamento estratégico que envolva, coordenadamente, capacitação das equipes, ações de vigilância da saúde, com priorização para a busca ativa, diagnóstico, tratamento e acompanhamento de casos $^{16}$.

Outro fato que pode estar relacionado a esse aumento do número de casos novos no ano de 2008 diz respeito a implementação do Plano Nacional de Controle da Hanseníase (PNCH) que ocorreu a partir do referido ano. Com o $\mathrm{PCNH}$, os serviços de saúde, dentre outras medidas, adotaram como meta a detecção mais intensa do número de casos novos menores de 15 anos, além de avaliação de incapacidades e exames de contato, o que acarretou em um aumento na busca ativa e consequentemente, um maior número de casos novos detectados ${ }^{17}$.

No presente estudo, o coeficiente de detecção de $\mathrm{CN}$ em crianças menores de 15 anos apresentou uma tendência de declínio, sugerindo uma diminuição de fontes de transmissibilidade e um pequeno avanço na eliminação da hanseníase na região. Tal achado vai de encontro a uma pesquisa realizada no país, que analisou a tendência temporal da hanseníase nessa população entre 2001 e 2016, evidenciando uma redução significativa do número de casos novos em menores de 15 anos ao longo série histórica, que pode estar associado ao avanço dos serviços de saúde na vigilância e controle da doença ${ }^{18}$.

No entanto, a ocorrência da hanseníase nessa faixa etária reafirma a manutenção de focos ativos de transmissão, com exposição precoce ao M. leprae. Como possíveis explicações para essa situação, inserem-se a dificuldade para o diagnóstico clínico, que se acentua nesse período da vida, o estigma relacionado à doença e a fragilidade do processo de promoção e educação em saúde nos territórios ${ }^{19}$.

Quanto ao coeficiente de detecção de GIF 2 em CN, verificou-se uma elevação significativa das taxas que consequentemente repercute negativamente na qualidade de vida e na saúde da população atingida. Esta situação demanda maior atenção dos serviços de saúde da região, tendo em vista que uma assistência integral ofertada pela atenção básica propicia aos pacientes hansênicos uma identificação precoce de alterações causadas principalmente pela lesão neural que limita as atividades diárias do paciente e repercute diretamente na sua funcionalidade, por isso a importância de analisar a incapacidade no período de tratamento da doença ${ }^{20}$.

Apesar dos esforços para o diagnóstico precoce, um contingente considerável de pessoas ainda é acometido por incapacidades físicas causadas pela hanseníase e observa-se, entre outros fatores, a dificuldade de acesso aos serviços de saúde, profissionais pouco qualificados para realização do diagnóstico da doença, ou que realizam consultas de curta duração, sem avaliar minuciosamente toda a superfície corporal estão entre os fatores que contribuem para o diagnóstico tardio da doença, resultando em atraso no início do tratamento e maior chance de evoluir com algum grau de incapacidade física ${ }^{21}$.

Assim, apesar do impacto da doença aos pacientes acometidos e considerando que o tratamento é realizado no sistema público de saúde, a hanseníase continua sendo um desafio enfrentado pela Atenção Primária devido aos fatores citados anteriormente e além disso, por ter seu tratamento negligenciado em muitas situações. Desse modo, faz-se necessário incentivar campanhas de esclarecimento à população quanto aos sinais e sintomas da doença, capacitar os profissionais de saúde 
para que possam diagnosticar e tratar os casos de hanseníase, como também realizar ações de promoção da saúde e assegurar estrutura adequada às unidades de saúde, a fim de garantir o acesso da população aos serviços 22,23 .

A identificação de GIF 2 no sujeito hansênico, também representa um marcador indireto de endemia oculta, permitindo estimar a prevalência mais próxima do cenário real da doença em um dado território ${ }^{24}$. Essa análise torna-se importante no sentido de definir estratégias locais com bases mais realísticas, condição essencial para a região de saúde de Crateús. Outra importante questão remete à necessidade de seguimento das rotinas estabelecidas pelas diretrizes nacionais para realização e registro adequado do escore Olho, Mão e Pé (OMP), minimamente no diagnóstico, ao longo do tratamento e durante a alta. $\mathrm{O}$ alto percentual de casos com ausência de informações referente ao GIF na alta impossibilitou, neste estudo, análise mais consistente desse importante indicador para toda a $15^{a}$ região ${ }^{21}$.

A maior ocorrência de casos multibacilares $(55,9 \%)$ em relação ao total de casos, com tendência de crescimento significativo na região do estudo, reforça a magnitude epidemiológica da endemia. Segundo Quaresma et al. ${ }^{25}$, as tendências da hanseníase, quando analisadas a respeito de seu caráter endêmico, podem ser observadas a partir do total de pessoas com infecção paucibacilar ou multibacilar, o que também reflete na maneira como está acontecendo o diagnóstico dos casos, se a maioria está acontecendo quando o paciente apresenta a forma multibacilar, supostamente está ocorrendo um diagnóstico tardio.

É nesta perspectiva que o presente artigo se direciona para o estado do Ceará, apontado por Goya et al. ${ }^{26}$ como "um solo fértil para o desenvolvimento de estudos da regionalização da saúde", cuja trajetória e experiência de constituição de Redes de Atenção à Saúde (RAS) em perspectiva regional antecedem à regulamentação federal do $\operatorname{SUS}^{27,28}$. Dessa forma, analisar a hanseníase e seus indicadores epidemiológicos ao longo de uma série temporal, em uma Coordenadoria Regional de Saúde, corrobora para uma reflexão acerca das fragilidades e potencialidades dessa forma de organização da região em estudo, possibilitando a criação de ações e reestruturação de serviços que visem o controle da doença, bem como a efetividade da assistência ao paciente com hanseníase em vista da qualidade do cuidado a essa população.

Além do mais, um fator contraditório nos achados do presente estudo foi o grande número de casos com a forma indeterminada da doença, enquanto o predomínio é de pacientes multibacilares. Tal fato pode estar relacionado a falta de capacitação dos profissionais no momento de determinar qual a forma clínica de hanseníase o paciente apresenta e assim, classificarem como indeterminada na grande maioria das fichas de notificação.

Nesse contexto, o estudo pode subsidiar profissionais de saúde na construção de uma prática integral e efetiva a pacientes acometidos por hanseníase, ao passo que a pesquisa possibilita uma análise de aspectos relacionados com a hanseníase e sua tendência ao longo de 15 anos em uma região de saúde do estado do Ceará. É fundamental que enfermeiros e a equipe de saúde considerem os determinantes sociais de saúde - tais como renda, trabalho, acesso a serviços, escolarização, entre outros - na prestação de cuidados, sobretudo em doenças que são historicamente negligenciadas como é o caso da hanseníase. Isso pode qualificar o cuidado e auxiliar na indicação de políticas públicas para o enfrentamento de doenças exacerbadas pela desigualdade social.

O estudo apresenta limitações relativas à utilização de bases de dados secundários, considerando-se a não completitude e as inconsistências para algumas variáveis. Entretanto, a incorporação do banco de dados do estado em uma série histórica de 15 anos, aliada à necessidade de estudos com essa abordagem na região de saúde de Crateús, justifica a sua utilização.

\section{CONCLUSÃO}

A análise da tendência através do método de regressão por pontos de inflexão foi útil para mostrar as alterações de acréscimo, redução ou estabilidade dos indicadores ao longo do tempo de estudo, encontrando assim, redução no número de hanseníase em menores de 15 anos e aumento do número de casos multibacilares e com grau 2 de incapacidade física.

O estudo demonstra a necessidade da criação e efetivação de estratégias que proporcionem a 
diminuição dos determinantes que contribuem para a disseminação da hanseníase na $15^{\mathrm{a}}$ CRES, tais como: melhoria das condições de salubridade da população, metas para o avanço de políticas públicas que favoreçam a saúde e os fatores sociais, bem como desenvolvimento de mutirões para detecção precoce.

Portanto, a qualificação das ações de controle desenvolvidas pelos serviços na rede do SUS deve ser sempre buscada. Sugere-se que novas pesquisas sejam realizadas em áreas que apresentem alta endemicidade, com o objetivo de analisar a cadeia de transmissão da doença, bem como a baixa resolutividade dos serviços de saúde primária na prevenção, diagnóstico e acompanhamento terapêutico. Sugere-se o desenvolvimento de pesquisas operacionais em saúde como meio estratégico para o fortalecimento das redes de atenção, em especial ao se considerar a complexidade da eliminação como meta de controle em saúde pública para a hanseníase.

\section{Participação dos autores:}

ALM Feitosa trabalhou na concepção e projeto, análise e interpretação dos dados. FW Dourado Júnior trabalhou na redação do texto. CMGD Florêncio trabalhou na concepção do projeto e revisão crítica relevante do conteúdo intelectual e aprovação final da versão a ser publicada.

\section{REFERÊNCIAS}

1. Neiva RJ. Perspectivas históricas do diagnóstico e tratamento da hanseníase.Sau. \& Transf. Soc 2016; $7(1): 88-97$.

2. World Health Organization. Estratégia Global Aprimorada para Redução Adicional da Carga da Hanseníase (Período do Plano: 2011-2015). Brasília: Organização Pan-Americana da Saúde; 2010 p. 1-48.

3. Brasil. Ministério da Saúde. Caracterização da Situação epidemiológica da hanseníase e diferenças por sexo, Brasil, 2012- 2016. Boletim epidemiológico 2018; 22 (1):1-55.

4. Penna M, Oliveira M, Penna G. The epidemiological behaviour of leprosy in Brazil. 2009. Lepr Rev 2009; $80(2): 332-344$.

5. Ribeiro MDA, Silva JCA, Oliveira SB. Estudo epidemiológico da hanseníase no Brasil: reflexão sobre as metas de eliminação. Rev Panam Salud Publica 2018; 42:1-7.
6. Sousa GS de, Silva Rodrigo LF da, Xavier MB. Hanseníase e Atenção Primária à Saúde: uma avaliação de estrutura do programa. Saúde debate 2017; 41(112): 230-242.

7. Nogueira PSF, Marques MB, Coutinho JFV, Maia JC, Silva MJD, Moura ERF. Factors associated with the functional capacity of older adults with leprosy. Rev. Bras. Enferm. 2017;70(4):711-718. doi:10.1590/0034-7167-2017-0091.

8. Reis MC, Raposo MT, Alencar CHM de, Ramos Júnior AM.Incapacidades físicas em pessoas que concluíram a poliquimioterapia para hanseníase em Vitória da Conquista, Bahia, Brasil. Acta Fisiatr 2018;25(2):78-85.

9. Oliveira KS de, Moraes MA, Deschutter EJ, Silva AJ da,Zilly A, Sobrinho RAS. Hanseníase em países fronteiriços na América do Sul: um estudo ecológico. Cogitare enferm 2019, 24:1-12.

10. Instituto Brasileiro de Geografia e Estatística (IBGE). Conheça cidades e estados do Brasil. [Internet]. 2020 [cited 2020 mai 26]; Available from: https://cidades. ibge.gov.br/brasil/ce/panorama.

11. Ceará.Secretaria Estadual de Saúde. Coordenadorias Regionais de Saúde. [Internet]. 2018. [cited 2020 mai 26]. Disponível em: https://www.saude.ce.gov.br/2018/07/03/ coordenadorias-regionais/.

12. Brasil. Ministério da Saúde. Diretrizes para vigilância, atenção e eliminação da hanseníase como problema de saúde pública:manual técnico-operacional. Brasília: MS; 2016.

13. Atlas do Desenvolvimento Humano no Brasil. Consulta do Índice de Desenvolvimento Humano. [Internet]. 2010 [cited 2020 Jul 19]; Available from: http://www. atlasbrasil.org.br/2013/pt/consulta/

14. Chaves EC, Costa SV, Reis Flores RLdos, Neves EOS das. Índice de carência social e hanseníase no estado do Pará em 2013: análise espacial. Epidemiol. Serv. Saúde 2017; 26(4): 807-816.

15. E-Gestor Atenção Básica. Informação e Gestão da Atenção Básica. [Internet]. 2020 [cited 2020 mai 26]; Disponível em: https://egestorab.saude.gov.br/paginas/acessoPublico/ relatorios/relHistoricoCoberturaAB.xhtml

16. Pereira WMM, Oliveira SS de, Sadeck LWR, Narvaes I da S, Adami M, Garcez LM. Hanseníase em metrópole da Amazônia brasileira: cobertura de atenção básica à saúde e sua relação com o perfil clínico e a distribuição espaço-temporal da doença em Belém, estado do Pará, Brasil, de 2006 a 2015. Rev Pan-Amaz Saude [Internet]. 2019 Mar [citado 2020 Maio 26]; 10: e201900069. Disponível em: http://scielo.iec.gov.br/scielo.php?scrip$\mathrm{t}=\mathrm{sci}$ _arttext\&pid=S2176-62232019000100015\&lng=pt.

17. Brasil. Ministério da Saúde. Programa Nacional de Controle da Hanseníase: Relatório de Gestão maio de 2007 a dezembro de 2008. [Internet]. Brasília: MS; 2009 [cited 2020 jul 19]. Disponível em: http://bvsms.saude.gov.br/ bvs/publicacoes/programa_nacional_controle_hanseniase_relatorio_gestao_maio_2007_dezembro_2008.pdf 
18. Schneider PB, Freitas BHBM de. Tendência da hanseníase em menores de 15 anos no Brasil, 2001-2016. Cad. Saúde Pública [Internet]. 2018 [cited 2020 Mai 26]; 34(3): e00101817.Disponível em: http:// www.scielo.br/scielo.php?script $=$ sci_arttext\&pi$\mathrm{d}=$ S0102-311X2018000305014\&lng=en.

19. Souza EA de, Ferreira AF, Boigny RN, Alencar $\mathrm{CH}_{\text {, }}$ Heukelbach J, Martins-Melo FR. Hanseníase e gênero no Brasil: tendências em área endêmica da região Nordeste, 2001-2014. Rev. Saúde Pública [Internet]. 2018 [cited 2020 Maio 26]; 52: 20. Disponível em: http://www.scielo.br/scielo.php?script=sci_arttext\&pi$\mathrm{d}=$ S0034-89102018000100216\&lng =pt.

20. Martins M, Siqueira L, Zolli C, Amâncio N. Perfil dos pacientes cadastrados com hanseníase no centro clínico universitário em Patos de Minas. Rev. Med. (São Paulo) [Internet]. 2019 [citado 24 jul. 2020];98(5):304-8. Available from: https://www.revistas.usp.br/revistadc/ article/view/156412

21. Gracie R, Peixoto, JNB de, Soares, FBR dos, Hacker MAVB de. Analysis of the geographical distribution of cases of leprosy. Cien Saude Col [Internet].2017 [cited 2020 Mai 26];22(5):1695-1704. Disponível em: https:// www.ncbi.nlm.nih.gov/pubmed/2853 8938.

22. Portela NLC, Sousa PHL de, Melo LNL de. Fatores associados à incapacidade física de casos novos de hanseníase em Paço do Lumiar-MA, 2006-2015. Hygeia 2018;14(27):80 -88.

23. Morais JR, Furtado ÉZL. Grau de incapacidade física de pacientes com hanseníase. Rev enferm UFPE on line. [Internet]. 2018 [cited 2020 Mai 26]; 12(6):1625-32.
Disponível em: https://periodicos.ufpe.br/revistas/ revistaenfermagem/article/download/231049/29245.

24. Santana EMF, Brito KKG, Nogueira JA, Leabedal ODCP, Costa MML, Silva MA, et al. Deficiências e incapacidades na hanseníase: do diagnóstico à alta por cura. Rev. Eletr. Enf. [Internet]. 2018 [citado em 2020 Mai 26];20 (15):1-11. Disponível em: https://fi-admin.bvsalud. org/document/view/mjpfd.

25. Quaresma M do SM, Souza L da SC, Silva FBM da, Pontes CDN, Silva YJA da. Perfil clínico e epidemiológico dos pacientes portadores de hanseníase em uma unidade de referência no estado do Pará. REAS [Internet]. 10 jan.2019 [citado 19jul.2020]; (18):e269. Available from: https://www.acervomais. com.br/index.php/saude/article/view/269.

26. Goya N, Andrade LOM, Pontes RJS, Tarja FS. Regionalização da saúde: (in)visibilidade e (i)materialidade da universalidade e integralidade em saúde no trânsito de institucionalidades. Saúde Soc. 2016; 25(4):902-919.

27. Goya N, Andrade LOM, Pontes RJS, Tarja FS, Barreto ICHC. Percepção de gestores estaduais da saúde sobre o Contrato Organizativo da gestão Pública no Ceará, Brasil. Cien Saude Colet 2017; 22(4):1235-1244.

28. Mascarenhas GC, Shimizu HE. Os desafios do planejamento regional compartilhado à luz do Decreto No 7508 : um estudo de caso Sobral - Ceará. In: Silva RM, Jorge MSB, Silva Junior AG, organizadores. Planejamento, gestão e avaliação nas práticas de saúde. Fortaleza: EdUECE; 2016. p. 72-93. 